\title{
Invited Commentary on Growth and Development in Children with Gastrointestinal Parasitic Infections
}

\author{
Patricia Bryan $^{1} \cdot$ Rojelio Mejia ${ }^{1}$
}

Published online: 2 October 2015

(C) Springer International Publishing AG 2015

\begin{abstract}
Gastrointestinal parasites including soil-transmitted helminths (Ascaris lumbricoides, Trichuris trichiura, and hookworms-Necator americanus, Ancylostoma duodenale), Strongyloides stercoralis, and intestinal protozoa (Giardia lamblia, Cryptosporidium parvum/hominis, and Entamoeba histolytica) are among the most widespread infectious organisms disproportionately affecting children worldwide. Economically disadvantaged children living in resource limited areas of developing countries are at greatest risk for recurrent gastrointestinal parasitic infections. Infected children often suffer significant morbidity impacting growth outcomes, school attendance, and academic achievement. Consequently, morbidity imposed by disease burden associated with gastrointestinal parasitic infections can have long-term implications impeding educational success and future productivity furthering conditions of poverty. It follows that these infections are an important public health concern related to child health outcomes worldwide. The objective of this review is to highlight fundamental aspects of childhood growth and development essential for the investigation and further understanding of morbidity associated with gastrointestinal parasitic infections in children.
\end{abstract}

Keywords Preschool-age $\cdot$ School-age $\cdot$ Soil-transmitted helminths $\cdot$ Protozoa

Rojelio Mejia

rmejia@bcm.edu

Patricia Bryan

patricia.bryan@bcm.edu

1 National School of Tropical Medicine, Baylor College of Medicine, Houston, TX, USA

\section{Introduction}

Gastrointestinal (GI) parasitic infections disproportionately affect children and remain a serious public health concern worldwide, especially in developing countries $[1 \bullet, 2]$. Notably, children experience the highest GI parasite intensities, and greater numbers of children are infected with more than one species of GI parasite compared to adults [1•]. Soiltransmitted helminths (STHs) infect nearly one third of the global population, including over 270 million preschool-age children and over 600 million school-age children [2]. These infections are widely distributed in tropical and subtropical areas [1•], with the greatest numbers occurring in impoverished areas of developing countries lacking sanitation and clean water infrastructure [2]. Children are most commonly infected with soil-transmitted helmiths (STH) and are prone to coinfections with more than one species, namely Ascaris lumbricoides (roundworm) and Trichuris trichiura (whipworm) [3•]. In developing countries, children between 6 months and 5 years of age are most susceptible to infections with parasitic protozoa, with the most frequently reported infections caused by Giardia lamblia [4]. The prevalence of infections with Giardia lamblia among children in developing countries has been reported to be as high as $50 \%$ occurring at any given time [5] and more than $90 \%$ of children experiencing at least one episode of giardiasis before 12 months of age [6]. Although infections with Cryptosporidium spp. are uncommon in industrialized countries, they are widespread geographically and are a leading cause of waterborne outbreaks with the greatest number of reported cases occurring among children from 1 to 9 years of age [7]. The incidence of infection with Cryptosporidium spp. peaks in preschool-age children, with children often infected by 2 years of age [8•]. Additionally, infections with both STHs and parasitic protozoa, namely Giardia lamblia, have been increasingly 
recognized as commonly occurring together in children younger than 5 years of age [7].

Morbidity associated with childhood GI parasitic infections can be insidious and debilitating including malabsorption of micronutrients, reduced appetite, abdominal pain, impaired growth, and cognitive deficits [3•]. Subsequently, children are more likely to experience failure in attainment of full growth and developmental potential essential for long-term positive health outcomes. Central to evaluating health outcomes in children infected with GI parasitic infections is age at impact relative to infection. Because children experience greater vulnerability to any insults during critical stages of growth and development characteristically occurring during early childhood [9], age is an important factor in determining the degree and type of resulting effects that can be expected [3•].

Although substantial public health gains related to GI parasitic infections have been made over the past 2 decades [ $1 \bullet$, eliminating morbidity in children from these infections has yet to be accomplished [2]. Further gains can be acquired through a clearer understanding of the mechanisms involved in morbidity, especially the effects related to growth and cognitive outcomes which have enduring implications for future potential and productivity. Evidence of growth and developmental outcomes associated with GI parasitic infections along with fundamental components of early childhood growth and development are addressed in this review as contributing to a greater understanding of the morbidity associated with these infections in children.

\section{Growth Delays}

Children experience two important distinct periods of accelerated growth (growth spurt), which are equally vital to child health outcomes. The first important period of growth occurs during the first 2 years of life. During this time, adequate nutritional intake is necessary in order to meet the high energy demands for optimal growth.

Growth during childhood is an important health indicator. Accordingly, growth monitoring throughout childhood is critical for early identification and prevention of poor health outcomes. Similarly, impaired growth during childhood is considered a surrogate marker for child health consequent to its association with impaired cognitive developmental potential [9]. Additionally, growth deficits, such as stunting, are associated with adverse long-term consequences, further highlighting the importance of growth monitoring during childhood [10]. The early identification of growth impairment in children living in endemic areas also allows for vital opportunities in recuperation of growth having a significant positive impact on long-term health outcomes.
Meaningful growth data for children infected with GI parasites involves a direct measure of linear growth over time [11] and comparison of this and other anthropometric indices with normal reference population values. Using Z-scores for such comparisons enables classification of growth measurements that are outside of normal values or average percentile ranges [11]. Expressly important, is the use of standardized methods for all growth parameter measurements. Assessment of growth parameters and anthropometric indices are important for evaluation of health outcomes in children living in areas where infections with GI parasites are endemic. Notably, children experiencing deficits in weight-for-height typically have an average for age appearance [11]. Often, those children who are also at risk for being infected with GI parasitic infections are unrecognized; consequently, missing vital opportunities for recuperating growth. In addition to evaluating linear growth, head circumference is an especially important growth parameter in young children based on the association with cognitive development [9]. Measures of postnatal head circumference between 9 months and 9 years of age reflect brain growth and have been linked to performance on tests evaluating intelligence in school-age children [12]. Evidence also suggests that maximizing growth during infancy and childhood is critical for the attainment of peak cognitive capacity in adulthood [12].

\section{Early Child Development}

A clear definition of developmental domains associated with GI parasitic infections can enable a better understanding of implications on child developmental outcomes [13]. Additionally, failure to account for external influences can substantially impede clear identification of developmental domains being affected [14]. Moreover, children between 12 and 24 months of age are especially vulnerable to insults from GI parasitic infections owing to critical stages of growth and development characteristic of this age, including rapid brain growth $[11,14]$. During this time, children infected with GI parasites are at risk for disruption of developmental processess $[11,14]$. Early childhood development is also comprised of specific domains involving attainment of functional skills and competencies. These include sensor-motor, cognitivelanguage, and social-emotional function [9]. Acquisition of skills and competence through developmental domains is foundational for future academic success [14]. Notably, school readiness and academic success are influenced by competence across all developmental domains. Any disruptions in progression through these early childhood developmental domains can impede competence at time of insult, and subsequently influence competence in future development [9], which can potentially impact long-term educational success. 


\section{Preschool Age Children}

Early childhood is marked by critical stages of growth and cognitive development, which involve attainment of skills important to cognitive ability acquired through competence in developmental domains [14]. During these formative years, children experience the greatest vulnerability to insults from GI parasitic infections and even minor disruptions can have long-term implications for growth and cognitive developmental outcomes [14]. Current evidence confirms that GI parasitic infections are common in younger children living in endemic areas [15]. This age coincides with normal progression through developmental milestones characteristic of early childhood, such as exploration of the environment through touch and taste as well as increasingly becoming more mobile. Although such behavior is essential to early childhood development, this predisposes younger children to greater exposure to GI parasites in the environment. It is not surprising that infections with STHs, namely A. lumbricoides and $T$. trichiura, become established during the first 2 years of life [3•]. Recent prospective data supports a greater focus on younger age groups at risk for GI parasitic infections. These prospective findings on children in Kenya indicated that STH infection by 2 years of age was associated with lower growth attainment in terms of length and head circumference relative to WHO standards [7]. Significant growth deficits, such as stunting, among children living in endemic areas commonly occur between 6 months and 2 years of age and overlap when STH infections become established [16]. Upon evaluating STH infections individually, infection with hookworm in 2 year olds was associated with below-average growth in length and head circumference; infection with Ascaris lumbricoides by 12 to 18 months was associated with decreased z-scores for all anthropometric indices at these ages [7]. Furthermore, infection with Strongyloides stercoralis by 30 months of age was associated with lower head circumference measures at this age [7]. When examining GI parasitic infections, as a group versus species-specific infections, parasitized children were found to have lower growth measures for height, weight, and upper arm circumference compared to noninfected children [17]. Also, coinfections with STH in this young age group have been associated with greater impairment in linear growth compared to children with a single species infection and noninfected children [7].

Of parasitic protozoa infections, recent evidence indicated that asymptomatic infections with Giardia lamblia in children younger than 5 years of age as well as infections with Cryptosporidium parvum/hominis combined and separate infections [18] were associated with stunting, possibly indicating that a chronic Giardia lamblia carrier state can occur at a young age [19]. Additionally, lasting effects of infection with Giardia lamblia or with more than one STH species in early childhood were associated with lower scores for measures of intelligence and social development respectfully, later in life during school age in children from a semi-urban slum in India [19]. This long-term effect was not observed for infection with Cryptosporidium spp. [18, 19]. When the social developmental domain was evaluated, neither infection with Giardia lamblia nor Cryptosporidium spp. was associated with scores for measure of social maturity [19]. Infections with Giardia lamblia have been reported to be associated with cognitive impairment and delayed psychomotor development in children younger than 5 years of age [7, 19]. In children as young as 3 years from a semi-urban slum in India, giardiasis, but not cryptosporidial diarrhea, was associated with lower scores on test assessing intelligence [19]. Similar results were not found when assessing social maturity in children infected with either Giardia lamblia or Cryptosporidium spp. [19].

\section{School Age Children}

Developmental milestones characteristic of school age can predispose children living in endemic areas to increased exposure to GI parasites. Children of this age spend much time playing outdoors, often in close contact with other children, near and around their home or homes of other children. Soil near homes in endemic areas is often contaminated, where open defecation may frequently occur together with approximating where STH eggs are transmitted [3•]. Prevalence rates of infection with GI parasites reflect that school age children are likely to harbor moderate-to-heavy STH infections, increasing their vulnerability to deficits in nutritional status [3•]. Consequently, children are at greater risk for infection. It follows that, in this age group, some children have a greater proclivity to infection than others imparted solely upon the characteristically incongruous attainment of competence across developmental domains and across the child age continuum.

GI parasitic infections in school age children have been widely investigated, and findings are consistently associated with growth and cognitive developmental deficits [3•]. Often, cognitive development is measured by school performance in this age group, with STH infections predominantly drawing focus. Infections with STHs, namely Ascaris lumbricoides [20] or Trichuris trichiura [21] as well as coinfections with both species, have been frequently associated with growth deficits and stunting in the school age population [3•]. The chronic nature of Trichuris trichiura has been implicated in the degree of growth deficit [17] during childhood.

Evidence suggests that children infected with GI parasites experience some impairment in cognitive ability based on psychometric tests evaluating different cognitive developmental domains [20, 22]. Infections with hookworm or Ascaris lumbricoides in rural Brazilian children have been associated with poor performance on tests evaluating cognitive 
functioning related to reasoning ability and general knowledge [20]. Among these children, coinfections with hookworm and Ascaris lumbricoides were also associated with poorer cognitive outcomes compared to children infected with a single STH infection [20]. In Filipino children, findings on infection with Ascaris lumbricoides of moderate-to-high intensity indicated an association with poor performance on tests of memory, and Trichuris trichiura was associated with poor performance on tests of verbal fluency regardless of infection intensity [22]. Similar to mediating effects on cognitive outcomes from anemia associated with hookworm infection, proximal influence of abdominal pain related to infections with Ascaris lumbricoides or Trichuris trichiura can notably impact attention and cognitive performance [23] in school age children. Unfortunately, complaints of abdominal discomfort from children in this age group can easily go unrecognized in association with GI parasitic infections. Pertaining to parasitic protozoa, infection with Entamoeba histolytica in school children did not have an effect on either verbal or nonverbal measures of general knowledge and intelligence, respectively [16]. However, lower scores for measures of intelligence were associated with Entamoeba histolytic-dysentery compared to children with dysentery of other etiologies [16]. Notably, evidence that etiology of diarrheal illness affects cognitive development differently may support reasons for some studies finding an association between diarrhea and neurodevelopment, while others do not [23].

\section{Conclusion}

Although the association between GI parasitic infections and child health outcomes has been widely investigated, the mechanisms involved in growth and cognitive deficits are not well established. Consequently, eliminating morbidity is not yet attainable, and GI parasitic infections in children remain a public health concern. Challenges also continue to arise for evaluation of child growth and cognitive outcome data, given the complexity and diversity of GI parasitic infections varying by country, community, and individual child [3•]. Further challenges are attributed to limited evidence on specific developmental domains affected by different GI parasite species infecting children. In addition, a lack of consistency in psychometric test used to assess cognitive development in younger children infected with GI parasites appears to reflect challenges inherent in choosing cognitive tests appropriate for measuring cognitive domains in preschool age children since there is no accepted psychometric test battery that can be applied across study populations and settings relative to children across the age continuum.

Aspects of growth and development addressed in this review provide potential directions for future research. One such direction involves a clear definition of cognitive domains associated with GI parasitic infections essential for understanding effects on cognitive development. Additionally, further investigation using rigorous design and methodology is necessary to produce evidence that will help to fully understand the mechanisms underlying the causal pathways of growth and cognitive developmental deficits in children infected with GI parasites. Within reach, a potential mechanism that deserves investigation to fill gaps in knowledge is the possible effect of decreased diversity of intestinal microbiota resulting from intestinal parasitic infections, which may have a significant impact on child health outcomes related to growth and cognitive development.

\section{Compliance with Ethics Guidelines}

Conflict of Interest Patricia Bryan declares that she has no conflict of interest.

Rojelio Mejia does consultant work for Lupin Pharmaceuticals, but this did not influence this paper.

Human and Animal Rights and Informed Consent This article does not contain any studies with human or animal subjects performed by any of the authors.

\section{References}

Papers of particular interest, published recently, have been highlighted as:

- Of importance

1. Pullan RL, Smith JL, Jasrasaria R, Brooker SJ. Global numbers of infection and disease burden of soil transmitted helminth infections in 2010. Parasites Vectors. 2014;7(37):1-19. Current global prevalence and estimates of public health burden of STH infections.

2. World Health Organization (WHO). Soil-transmitted helminth infections. Global distribution and prevalence. 2015. http://www. who.int/mediacentre/factsheets/fs366/en/.

3. Hall A, Hewitt G, Tuffrey V, de Silva N. A review and metaanalysis of the impact of intestinal worms on child growth and nutrition. Matern Child Nutr. 2008;4:118-236. Comprehensive and informative systematic review and meta-analysis of morbidity associated with intestinal parasites related to child growth and nutrition.

4. Simsek Z, Derek FY, Kurcer MA. Effect of Giardia infection on growth and psychomotor development in children aged $0-5$ years. J Trop Pediatr. 2004;50(2):90-3.

5. Savioli L, Smith H, Thompson A. Correlational between genotype of Giardia duodenalis and diarrhea. Int J Parasitol. 2006;32:229-31.

6. Ali SA, Hill DR. Giardia intestinalis. Curr Opin Infect Dis. 2003;16(5):203-8.

7. LeBeaud AD, Singer MN, McKibben M, Mungal P, Muchiri EM, McKibben E, et al. Parasitism in children aged three years and under: relationship between infection and growth in rural coastal Kenya. PLoS Negl Trop Dis. 2015;9(5), e0003721.

8. Kvalsvig J, Albonico M. Effects of geohelminth infections on neurological development. Handb Clin Neurol. 2013;114:1. Very 
informative and comprehensive discussion of the impacts of STH on neurological development in children.

9. Gale CR, O'Callaghan, Godfrey KM, Law CM, Martyn CN. Critical periods of brain growth and cognitive function in children. Brain. 2004;1-9.

10. Pendergast, Humphrey. The stunting syndrome in developing countries. Paediatr Int Child Health. 2014;34(4):250-65.

11. de Onis M, Yang H. Algorithms for converting estimates of child malnutrition based on the NCHS reference into estimates based on the WHO Child Growth Standards. BMC Pediatr. 2008;8(19):1-6.

12. Santos DN, Assis AMO, Bastos ACS, Santos LM, Santos CAST, Strina A, et al. Determinants of cognitive function in childhood: a cohort study in a middle income context. BMC Public Health. 2008;8(202).

13. Walker S, Wachs T, Gardner MJ, et al. Child development: risk factors for adverse outcomes in developing countries. Lancet. 2007;369:145-7.

14. Orden AB, Apezteguia MC, Clarmela ML, Molina NB, Pezzani BC, Rosa D, et al. Nutritional status in parasitized and nonparasitized children from two districts of Buenos Aires, Argentina. Am J Hum Biol. 2014;25(73): 79.

15. Gyorkos TW, Maheu-Giroux M, Casapia M, Joseph SA, CreedKanashiro H. Stunting and helminth infection in early preschoolage children in a resource-poor community in the Amazon lowlands of Peru. Trans R Soc Trop Med Hyg. 2011;105:204-8.

16. Berkman DS, Lescano AG, Gilman RH, Lopez SL, Black MM. Effects of stunting, diarrhoeal disease, and parasitic infection during infancy on cognition in late childhood: a follow-up study. Lancet. 2002;359:564-71.

17. Botero-Garces JH, Garcia-Montoya GM, Grisales-Patino D, Aguirre-Acevedo DC, Alvarez-Uribe MC. Giardia intestinalis and nutritional status in children participating in the complementary nutrition program, Antioquia, Columbia, May to October 2006. Rev Inst Med Trop Sao Paulo. 2009;51(3):155-62.

18. Molloy SF, Tanner CJ, Kirwan P, Asaolu SO, Smith HV, Nichols $\mathrm{RAB}$, et al. Sporadic Cryptosporidium infection in Nigerian children: risk factors with species identification. Epidemiol Infect. 2011;139:946-54.

19. Ajjampur SSR, Koshy B, Venkataramani M, Sarkar R, Joseph AA, Jacob KS, et al. Effect of Cryptosporidial and giardial diarrhea on social maturity, intelligence and physical growth in children in a semi-urban slum in south India. Ann Trop Paediatr. 2011;31:20512.

20. Albonico M, Allen H, Chitsulo L, Engels D, Gabrielli A-F, Savioli L. Controlling soil-transmitted helminthiasis in pre-school age children through preventive chemotherapy. PLoS Negl Trop Dis. 2008;2(3), e1126.

21. Ezeamama AE, Friedman JF, Acosta LP, Bellinger DC, Langdon GC, Manalo DL, et al. Helminth infection and cognitive impairment among Filipino children. Am J Trop Med Hyg. 2005;72:5408.

22. Oria RB, Costa CMC, Lima AAM, Patrick PD, Guerrant RL. Semantic fluency: a sensitive marker for cognitive impairment in children with heavy diarrhea burdents? Med Hypotheses. 2009;73: 682-9.

23. Jardim-Botelho A, Raff S, de Avila R, Hoffman HJ, Diemert DJ, Correa-Oliveira R, et al. Hookworm, Ascaris lumbricoides infection and polyparasitism associated with poor cognitive performance in Brazilian schoolchildren. Trop Med Int Health. 2008;13(8):9941004. 